\title{
MORPHOLOGICAL CHARACTERIZATION OF ISOLATES OF PHOMOPSIS VEXANS OF BANGLADESH
}

\author{
M M Islam ${ }^{1}$, M Asaduzzaman ${ }^{2}$, M E Hoque ${ }^{3}$ and M B Meah ${ }^{4}$
}

\begin{abstract}
Forty four isolates of Phomopsis vexans from different eggplant cultivars collected from core eggplant growing regions of Bangladesh which were characterized using mycological characteristics. Variation exists among the isolates of Phomopsis vexans of Bangladesh covering two types of farm having two ecosystems and the isolates were grouped into five distinct groups based on their cultural properties. The highest sized $\alpha$ conidia were observed in group -1 and the lowest size in-group -2 . The highest sized $\beta$ conidia were recorded in group -5 and the lowest in-group -2 . The highest pycnidial size noted in-group -3 and the smallest sized pycnidia were recorded in group -1 .
\end{abstract}

Key words: Morphology, Phomopsis vexans, eggplant.

\section{INTRODUCTION}

Eggplant suffers from many diseases caused by fungi, bacteria, virus, nematode and mycoplasma. Of them Phomopsis fruit rot of eggplant caused by the fungus Phomopsis vexans (Sacc. and Syd) Harter is a serious disease which attacks all above ground parts of the plant. It is mentionable damaging to the crop and is a threat particularly in Kharif season and late crop in winter season. Halsted first described the organigm in the United States in 1892 as Phoma solani Halst. Since the name had been used for another fungus it was changed to Phoma vexans by Saccardo and Sydow in 1899. Harter transfered it to Phomopsis vexans (Sacc. and Syd.) Harter (Walker, 1952). Sugha et al. (2002) have reported that alpha and beta are two forms of the same conidium. Phomopsis vexans produces only one type of conidia in its pycnidia, which are hyaline, one celled, sub-cylindrical and 5-9 x 2-2.8 $\mu$ in size during summer months, which gradually changed into the beta form. Inoculation of host plants with beta conidia caused intraveinal necrosis, which progressed towards the leaf base and resulted in premature defoliation, thus indicating their role in pathogenesis. Isolations from such leaves produced pycnidia with alpha conidia at $25^{\circ} \mathrm{C}$ and beta conidia at $16^{\circ} \mathrm{C}$. Singh (1992) has reported that, the perfect or sexual stage of the pathogen is Diaporthe vexans. On the leaves, the pycnidia are 60 to $200 \mu$ in diameter and on fruits they are 120 to $350 \mu$. They are globose to irregular with 20 to $50 \mu$ wide ostiole. The conidiophores are hyaline, 10 to $16 \mu$ long. Conidia are hyaline, one-celled, sub-cylindrical, 5-9 x 2-2.9 $\mu$ in size. Another form of conidia, the stylospores ( $\beta$ conidia) are filiform, curved, hyaline and aseptate. These spores do not germinate; they are 20$30 \times 0.5-1.0 \mu$ in size. Information about the morphology of Phomopsis vexans in Bangladesh is very limited. Therefore, the present research work was undertaken to identify the morphological variability of Phomopsis vexans isolates existing in Bangladesh.

\section{MATERIALS AND METHODS}

Samples were collected from plants affected by Phomopsis blight/fruit rot of eggplant and showing symptoms on leaves, stems, fruits and seeds. The isolates came from 23 strategic localities in Bangladesh, according to 9 Agro-Ecological Zones (AEZ) covering two types of

\footnotetext{
${ }^{1}$ SO, Soil Science Division, BARI, Gazipur

${ }^{2}$ Executive Director, Bangladesh Science Foundation, Uttara, Dhaka-1230.

${ }^{3}$ Upazilla Agriculture Officer, Fulpur, Mymensingh.

${ }^{4}$ Professor, Department of Plant Pathology, Bangladesh Agricultural University, Mymensingh
} 
farm categories viz. experimental farms and farmers field having two ecosystems i.e. rainfed and irrigated field (Table 1). The samples were plucked and placed in polythene bag in airtight condition, later kept in the refrigerator for overnight for further study. The visible signs and symptoms were recorded in details and then photographs were also taken showing different stages of development of Phomopsis fruit rot, photographs were also taken from heavily infested eggplant plot of some areas. Temporary slides were prepared out of the diseased samples and examined under microscope for pathogenic structures.

Diseased fruit, stem, leaf and seed samples with typical symptoms were selected and washed separately under running tap water to remove dust particles and then air dried. The infected fruits were surface disinfected by flaming in spirit lamp following soaking with cotton swab (70\% alcohol). Small pieces $(2-3 \mathrm{~mm})$ of fruit tissues at the junction of diseased and healthy portion were cut with the help of sterilized scalpel from eggplant fruit for the preparation of inocula. Inocula were placed on Potato Dextrose Agar (PDA) plates (following tissue planting method) acidified with one drop of 5\% lactic acid and then the petridishes were sealed with Para film and incubated at room temperature $\left(25 \pm 2^{0} \mathrm{C}\right)$ for seven days to allow the pathogen to grow. After incubation, white mycelia were formed. Similar growth was observed on almost all inocula. The organism was aseptically transferred to several fresh PDA plates and incubated at room temperature from when sub-cultures were made for the purification by transferring fungal block with conidia with the help of sterilized block cutter $(5 \mathrm{~mm})$. It was sub-cultured several time to avoid any contamination and then purified and multiplied on PDA. Following the available literatures (Punithalingam and Holliday 1972).The organism was identified through microscopic observation.

Table 1. Isolates of Phomopsis vexans (PV) used for morphological observations.

\begin{tabular}{|c|c|c|c|c|c|c|}
\hline $\begin{array}{l}\text { Isolate } \\
\text { code }\end{array}$ & $\begin{array}{c}\text { Host } \\
\text { (Eggplant } \\
\text { cultivars) } \\
\end{array}$ & $\begin{array}{c}\text { Plant } \\
\text { part(s) }\end{array}$ & Ecosystem & $\begin{array}{c}\text { Farm } \\
\text { category }\end{array}$ & $\begin{array}{c}\text { Geographic } \\
\text { location }\end{array}$ & Collector \\
\hline 1 & Uttara & Fruit & Irrigated & $\begin{array}{c}\text { Experimental } \\
\text { farm }\end{array}$ & $\begin{array}{c}\text { BAU } \\
\text { Mymensingh }\end{array}$ & M.Islam \\
\hline 2 & Laffa & Stem & $\begin{array}{l}\text { Rainfed } \\
\text { highland }\end{array}$ & $\begin{array}{l}\text { Farmer's } \\
\text { field }\end{array}$ & $\begin{array}{l}\text { Nandina } \\
\text { Jamalpur }\end{array}$ & R. Islam \\
\hline 3 & Talbegun & Leaf & $\begin{array}{l}\text { Rainfed } \\
\text { highland }\end{array}$ & $\begin{array}{c}\text { Farmer's } \\
\text { field }\end{array}$ & $\begin{array}{l}\text { Mireshawry } \\
\text { Chittagong }\end{array}$ & R.Islam \\
\hline 4 & Laffa & Fruit & $\begin{array}{l}\text { Rainfed } \\
\text { lowland }\end{array}$ & $\begin{array}{c}\text { Farmer's } \\
\text { field }\end{array}$ & Sherpur & Y.Ali \\
\hline 5 & Zhumki & Fruit & $\begin{array}{l}\text { Rainfed } \\
\text { lowland }\end{array}$ & $\begin{array}{l}\text { Farmer's } \\
\text { field }\end{array}$ & $\begin{array}{l}\text { Nandina } \\
\text { Jamalpur }\end{array}$ & Y.Ali \\
\hline 6 & Kaikka & Seed & $\begin{array}{l}\text { Rainfed } \\
\text { lowland }\end{array}$ & $\begin{array}{c}\text { Farmer's } \\
\text { field }\end{array}$ & $\begin{array}{l}\text { Satpaikka } \\
\text { Sherpur }\end{array}$ & R.Islam \\
\hline 7 & Zhumki & Fruit & $\begin{array}{l}\text { Rainfed } \\
\text { lowland }\end{array}$ & $\begin{array}{c}\text { Farmer's } \\
\text { field }\end{array}$ & $\begin{array}{l}\text { Nandina } \\
\text { Jamalpur }\end{array}$ & Y.Ali \\
\hline 8 & Uttara & Fruit & $\begin{array}{l}\text { Irrigated } \\
\text { highland }\end{array}$ & $\begin{array}{l}\text { Experimental } \\
\text { farm }\end{array}$ & $\begin{array}{c}\text { BAU } \\
\text { Mymensingh }\end{array}$ & M.Islam \\
\hline 9 & Dohazari & Stem & $\begin{array}{l}\text { Rainfed } \\
\text { highland }\end{array}$ & $\begin{array}{l}\text { Farmer's } \\
\text { field }\end{array}$ & $\begin{array}{c}\text { Dohazari } \\
\text { Chittagong }\end{array}$ & R.Islam \\
\hline 10 & Singnath & Fruit & $\begin{array}{l}\text { Rainfed } \\
\text { lowland }\end{array}$ & $\begin{array}{l}\text { Farmer's } \\
\text { field }\end{array}$ & $\begin{array}{l}\text { Chandina } \\
\text { Comilla }\end{array}$ & M.Islam \\
\hline 11 & Khatkhatia & fruit & $\begin{array}{l}\text { Rainfed } \\
\text { lowland }\end{array}$ & $\begin{array}{l}\text { Farmer's } \\
\text { field }\end{array}$ & $\begin{array}{c}\text { Bhurungamari } \\
\text { Lalmonirhat }\end{array}$ & R.Islam \\
\hline 12 & Uttara & Fruit & $\begin{array}{l}\text { Rainfed } \\
\text { lowland }\end{array}$ & $\begin{array}{c}\text { Farmer's } \\
\text { field }\end{array}$ & $\begin{array}{l}\text { Shombugonj } \\
\text { Mymensingh }\end{array}$ & M.Islam \\
\hline
\end{tabular}




\begin{tabular}{|c|c|c|c|c|c|c|}
\hline 13 & Dohazari & Fruit & $\begin{array}{l}\text { Rainfed } \\
\text { highland }\end{array}$ & $\begin{array}{l}\text { Farmer's } \\
\text { field }\end{array}$ & $\begin{array}{c}\text { Dohazari } \\
\text { Chittagong }\end{array}$ & M.Islam \\
\hline 14 & Rupgonj L & Stem & $\begin{array}{l}\text { Rainfed } \\
\text { lowland }\end{array}$ & $\begin{array}{c}\text { Farmer's } \\
\text { field }\end{array}$ & $\begin{array}{c}\text { Rupgonj } \\
\text { Narayangonj }\end{array}$ & M.Islam \\
\hline 15 & Singnath & Fruit & $\begin{array}{l}\text { Rainfed } \\
\text { highland }\end{array}$ & $\begin{array}{c}\text { Farmer's } \\
\text { field }\end{array}$ & $\begin{array}{l}\text { Chandina } \\
\text { Comilla }\end{array}$ & M.Islam \\
\hline 16 & Khatkhatia & Seed & $\begin{array}{l}\text { Rainfed } \\
\text { lowland }\end{array}$ & $\begin{array}{c}\text { Farmer's } \\
\text { field }\end{array}$ & $\begin{array}{l}\text { Nageshawri } \\
\text { Lalmonirhat }\end{array}$ & R. Islam \\
\hline 17 & Kaikka & Stem & $\begin{array}{l}\text { Rainfed } \\
\text { lowland }\end{array}$ & $\begin{array}{c}\text { Farmer's } \\
\text { field }\end{array}$ & Sherpur & R.Islam \\
\hline 18 & Kaikka & Fruit & $\begin{array}{l}\text { Rainfed } \\
\text { lowland }\end{array}$ & $\begin{array}{c}\text { Farmer's } \\
\text { field }\end{array}$ & Sherpur & R.Islam \\
\hline 19 & Khatkhatia & Fruit & $\begin{array}{l}\text { Rainfed } \\
\text { highland }\end{array}$ & $\begin{array}{c}\text { Farmer's } \\
\text { field }\end{array}$ & $\begin{array}{c}\text { Bhurungamari } \\
\text { Lalmonirhat }\end{array}$ & R.Islam \\
\hline 20 & Dohazari & Seed & $\begin{array}{l}\text { Rainfed } \\
\text { lowland }\end{array}$ & $\begin{array}{l}\text { Farmer's } \\
\text { field }\end{array}$ & $\begin{array}{l}\text { Nageshawri } \\
\text { Lalmonirhat }\end{array}$ & R.Islam \\
\hline 21 & Indigo & Fruit & $\begin{array}{l}\text { Irrigated } \\
\text { highland }\end{array}$ & $\begin{array}{l}\text { Experimental } \\
\text { farm }\end{array}$ & $\begin{array}{c}\text { BAU } \\
\text { Mymensingh }\end{array}$ & M.Islam \\
\hline 22 & Dohazari & Stem & $\begin{array}{l}\text { Rainfed } \\
\text { lowland }\end{array}$ & $\begin{array}{l}\text { Farmer's } \\
\text { field }\end{array}$ & $\begin{array}{c}\text { Dohazari } \\
\text { Chittagong }\end{array}$ & M.Islam \\
\hline 23 & China & Fruit & $\begin{array}{l}\text { Irrigated } \\
\text { highland }\end{array}$ & $\begin{array}{l}\text { Farmer's } \\
\text { field }\end{array}$ & $\begin{array}{c}\text { Paba } \\
\text { Rajshahi }\end{array}$ & y. Ali \\
\hline 24 & Thamba & Fruit & $\begin{array}{l}\text { Rainfed } \\
\text { highland }\end{array}$ & $\begin{array}{c}\text { Farmer's } \\
\text { field }\end{array}$ & $\begin{array}{c}\text { Sherpur } \\
\text { Bogra }\end{array}$ & Y. Ali \\
\hline 25 & Modhukhali & Fruit & $\begin{array}{l}\text { Rainfed } \\
\text { lowland }\end{array}$ & $\begin{array}{l}\text { Farmer's } \\
\text { field }\end{array}$ & $\begin{array}{l}\text { Modhukhali } \\
\text { Faridpur }\end{array}$ & Howlader \\
\hline 26 & Zhumki & Fruit & $\begin{array}{l}\text { Rainfed } \\
\text { lowland }\end{array}$ & $\begin{array}{c}\text { Farmer's } \\
\text { field }\end{array}$ & $\begin{array}{l}\text { Nandina } \\
\text { Jamalpur }\end{array}$ & Y.Ali \\
\hline 27 & Bijoy & Fruit & $\begin{array}{l}\text { Rainfed } \\
\text { lowland }\end{array}$ & $\begin{array}{c}\text { Farmer's } \\
\text { field }\end{array}$ & $\begin{array}{l}\text { Modhukhali } \\
\text { Faridpur }\end{array}$ & Howlader \\
\hline 28 & Dohazari & Fruit & $\begin{array}{l}\text { Rainfed } \\
\text { highland }\end{array}$ & $\begin{array}{l}\text { Farmer's } \\
\text { field }\end{array}$ & $\begin{array}{l}\text { Nageshari } \\
\text { Chittagong }\end{array}$ & R.Islam \\
\hline 29 & Talbegun & Stem & $\begin{array}{l}\text { Rainfed } \\
\text { highland }\end{array}$ & $\begin{array}{l}\text { Farmer's } \\
\text { field }\end{array}$ & Lalmonirhat & R.Islam \\
\hline 30 & China oblong & Stem & $\begin{array}{l}\text { Irrigated } \\
\text { lowland }\end{array}$ & $\begin{array}{l}\text { Farmer's } \\
\text { field }\end{array}$ & $\begin{array}{c}\text { Paba } \\
\text { Rajshahi }\end{array}$ & M.Islam \\
\hline 31 & Laffa B & Fruit & $\begin{array}{l}\text { Rainfed } \\
\text { lowland }\end{array}$ & $\begin{array}{c}\text { Farmer's } \\
\text { field }\end{array}$ & $\begin{array}{c}\text { Rupgonj } \\
\text { Narayangonj }\end{array}$ & M.Islam \\
\hline 32 & Khatkhatia & Fruit & $\begin{array}{l}\text { Rainfed } \\
\text { highland }\end{array}$ & $\begin{array}{l}\text { Farmer's } \\
\text { field }\end{array}$ & Lalmonirhat & R.Islam \\
\hline 33 & Khatkhatia & Fruit & $\begin{array}{l}\text { Rainfed } \\
\text { lowland }\end{array}$ & $\begin{array}{l}\text { Farmer's } \\
\text { field }\end{array}$ & $\begin{array}{l}\text { Modhukhali } \\
\text { Faridpur }\end{array}$ & Y. Ali \\
\hline 34 & Jessore L & Seed & $\begin{array}{l}\text { Irrigated } \\
\text { highland }\end{array}$ & $\begin{array}{l}\text { Farmer's } \\
\text { field }\end{array}$ & $\begin{array}{c}\text { Monirampur } \\
\text { Jessore }\end{array}$ & Y. Ali \\
\hline 35 & Kaikka & Leaf & $\begin{array}{l}\text { Rainfed } \\
\text { lowland }\end{array}$ & $\begin{array}{l}\text { Farmer's } \\
\text { field }\end{array}$ & $\begin{array}{l}\text { Gaffargaoan } \\
\text { Mymensingh }\end{array}$ & M.Islam \\
\hline 36 & Khatkhatia & Fruit & $\begin{array}{l}\text { Rainfed } \\
\text { highland }\end{array}$ & $\begin{array}{c}\text { Farmer's } \\
\text { field }\end{array}$ & $\begin{array}{l}\text { Nageshari } \\
\text { Chittagong }\end{array}$ & R.Islam \\
\hline
\end{tabular}

Table Contd. 


\begin{tabular}{|c|c|c|c|c|c|c|}
\hline 37 & Singnath & Fruit & $\begin{array}{c}\text { Rainfed } \\
\text { lowland }\end{array}$ & $\begin{array}{c}\text { Farmer's } \\
\text { field }\end{array}$ & $\begin{array}{c}\text { Savar } \\
\text { Dhaka }\end{array}$ & M.Islam \\
\hline 38 & Jessore L & Stem & $\begin{array}{c}\text { Rainfed } \\
\text { lowland }\end{array}$ & $\begin{array}{c}\text { Farmer's } \\
\text { field }\end{array}$ & $\begin{array}{c}\text { Monirampur } \\
\text { Jesssore }\end{array}$ & M.Islam \\
\hline 39 & Laffa & Stem & $\begin{array}{c}\text { Rainfed } \\
\text { lowland }\end{array}$ & $\begin{array}{c}\text { Farmer's } \\
\text { field }\end{array}$ & $\begin{array}{c}\text { Rupgonj } \\
\text { Narayangonj }\end{array}$ & Y. Ali \\
\hline 40 & Khatkhatia & Stem & $\begin{array}{c}\text { Rainfed } \\
\text { highland }\end{array}$ & $\begin{array}{c}\text { Farmer's } \\
\text { field }\end{array}$ & $\begin{array}{c}\text { Nageshari } \\
\text { Chittagong }\end{array}$ & R. Islam \\
\hline 41 & Laffa & Fruit & $\begin{array}{c}\text { Rainfed } \\
\text { highland }\end{array}$ & $\begin{array}{c}\text { Farmer's } \\
\text { field }\end{array}$ & Rangpur & R.Islam \\
\hline 42 & Soilabegun & Stem & $\begin{array}{c}\text { Rainfed } \\
\text { lowland }\end{array}$ & $\begin{array}{c}\text { Farmer's } \\
\text { field }\end{array}$ & Faridpur & Y.Ali \\
\hline 44 & Soilabegun & Fruit & $\begin{array}{c}\text { Rainfed } \\
\text { lowland }\end{array}$ & $\begin{array}{c}\text { Farmer's } \\
\text { field }\end{array}$ & Faridpur & M.Islam \\
\hline
\end{tabular}

The isolates, after confirmation were maintained in petri dishes on PDA media and transferred to new media once a month. To store for long periods, the isolates were maintained on PDA slants in test tube and kept at $4^{0} \mathrm{C}$. Morphological characterizations of 44 isolates of Phomopsis vexans collected during 2007-2008 were made. In all, 44 isolates were purified by picking up mycelial block culture and named according to the region of their collection. They were maintained on Acidic Potato Dextrose Agar (APDA) medium at $25 \pm 1^{0} \mathrm{C}$ for studying cultural and morphological characteristics. Based on the cultural properties, the isolates were grouped.

\section{RESULTS AND DISCUSSION}

Forty four isolates of Phomopsis vexans from different eggplant cultivars collected from core eggplant growing regions of Bangladesh were characterized and categorized into five groups based on their cultural properties like mycelial growth, colony colour, colony consistency, pycnidial distribution in the growing media, pycnidial size, spore size, and sporulation rate. The isolates $1,3,4,5,8,9,10,11,12,13,14,15,16,18,19,20,21,22,23,24,27,28,29,32,34$, $36,37,38,39,42,43$, and 44 were grouped in one (01), isolates 6,7 and 41 were in group (02), isolates 30, 33 and 35 were grouped in three, isolates 2 and 25 were grouped in four (04), and isolates 26, 31 and 40 were grouped in five (05) (Table 2).

Five groups of isolates distinctly differed on all the parameters evaluated with few exceptions. Mycelial growth was recorded the highest in group-5 followed by group-3, group 1 and group 4.

The lowest mycelial growth was observed in group-2. Colony colour slightly differed among the isolate groups from off-white to milk white. Only group-2 possessed grey /brownish colony. Colonies were embedded in group 1,2,3 \& 4 and slightly fluffy in group-5 but they differed on their consistency.

In case of spore size, the highest sized $\alpha$ conidia were observed in group -1 and the lowest size in-group -2. In case of $\beta$ conidia, the highest sized spore was recorded in group -5 and similarly the lowest in-group -2 . Sporulation was the highest in-group -1 followed by group -3, 4 and 2 . Isolate group -5 had the lowest sporulation (Table 3 ). In case of pycnidial distribution, group-1 
had huge number of pycnidia distributed densely in the media while presence of pycnidia was rare and submerged in group-5. Other isolate groups contained few to large number of pycnidia in the media. Pycnidial size differed significantly among the isolate groups. The highest pycnidial size noted in-group -3 . The smallest sized pycnidia were recorded in group -1 (Table $4)$.

Five groups of isolates of Phomopsis vexans distinctly differed on their cultural and morphological properties like mycelial growth, colony color, colony consistency, pycnidial distribution in the media, pycnidial size, spore ( $\alpha$ and $\beta)$ size and sporulation rate. Variability in the isolates might be due to the existence of physiological races of Phomopsis vexans in the environment. Previous studies support the findings of the present investigation (Islam et al., 2004; Islam et al., 2004 and Meah, 2003) who noted variation among 32 isolates of Phomopsis vexans isolated from diseased samples of 29 eggplant cultivars collected from 17 growing areas in Bangladesh. Meah (2003) also observed differential reactions even in the same cultivar against five groups of isolates in an in vitro cross inoculation on eggplant, which might be considered as the existence of physiological differences among the isolates. Islam et al. (2004) reported that $\alpha$ and $\beta$ conidia of Phomopsis vexans varied in size among 32 isolates, which exhibited the presence of physiological races in the environment. Islam et al. (2004) reported that the variation existed in the DNA banding patterns of P.vexans while they worked with 44 isolates collected from major eggplant growing areas of Bangladesh.

On Potato Dextrose Agar, the fungus P. vexans formed creamy white mycelial colony producing hyaline, single celled $\alpha$ conidia and elongated, hyaline, cylindrical or with characteristic band called $\beta$ conidia. The results are in conformity with those of Chinenye (1974).

In the present study, the length of conidia ranged for $\alpha$-conidia 4.6-7. $36 \mu$ and $\beta$ conidia 22.08 $55.2 \mu$ and the breadth ranged from $1.84-3.68 \mu$. (40x). The length of pycnidia ranged from 216.96- $589.86 \mu$ and the breadth ranged from $101.7-637.32 \mu$. $(10 \mathrm{x})$. The results do agree with the conidial measurement reported by Gratz (1942), Sharma and Agarwal (1973), Chinenye (1974), Singh (1992) and Punithalingam and Holliday (1972). It may be concluded that the 44 isolates were in five clusters by morphologically. 
Table 2. Five different groups of isolates of Phomopsis vexans with their cultural characters.

\begin{tabular}{|c|l|c|l|l|}
\hline Group & Ungrouped isolates & Colony color & Characteristics & Pycnidial properties \\
\hline 1 & $\begin{array}{l}1,3,4,5,8,9,10,11,12, \\
13,14,15,16,17,18,19, \\
20,21,22,23,24,27,28,29,32,34,3 \\
6,37,38,39,42,43,44\end{array}$ & Whitish & $\begin{array}{l}\text { Growth fluffy, } \\
\text { Colony compact and thick with } \\
\text { concentric ring }\end{array}$ & $\begin{array}{l}\text { Pycnidial structures/mats are relatively big } \\
\text { sized and sparsely distributed in lower } \\
\text { frequency }\end{array}$ \\
\hline 2 & $6,7,41$ & Whitish & $\begin{array}{l}\text { Growth embedded, Colony } \\
\text { compact and thick without } \\
\text { concentric ring }\end{array}$ & $\begin{array}{l}\text { Pycnidial structures/mats are small sized } \\
\text { and densely distributed in higher frequency }\end{array}$ \\
\hline 3 & $30,33,35$ & Blackish & $\begin{array}{l}\text { Growth embedded, Colony } \\
\text { compact and thick with slightly } \\
\text { concentric rings distinct. }\end{array}$ & $\begin{array}{l}\text { Pycnidial structures/mats are minute in size, } \\
\text { huge in number and densely distributed. }\end{array}$ \\
\hline 4 & 2,25 & Gray/brownish & $\begin{array}{l}\text { Growth fluffy, Colony compact } \\
\text { and thick with distinct concentric } \\
\text { ring }\end{array}$ & $\begin{array}{l}\text { Pycnidial structures/mats } \\
\text { are minute in size and densely distributed } \\
\text { in higher frequency }\end{array}$ \\
\hline 5 & $26,31,40$ & Milk white & $\begin{array}{l}\text { Growth slightly fluffy, Colony } \\
\text { loose and thin with concentric ring }\end{array}$ & $\begin{array}{l}\text { Pycnidial structures/mats are absent/rare } \\
\text { even after one month of inoculation in PDA } \\
\text { at 25 } \pm 2\end{array}$ \\
\hline
\end{tabular}


Table 3. Measurement of spores of Phomopsis vexans .

\begin{tabular}{|c|c|c|c|c|}
\hline \multirow{4}{*}{$\begin{array}{l}\text { Isolate } \\
\text { Group }\end{array}$} & \multicolumn{4}{|c|}{ Spore Measurement } \\
\hline & \multicolumn{4}{|c|}{$\begin{array}{c}\text { Spore size } \\
\text { (Length } \mathrm{x} \text { breadth) }\end{array}$} \\
\hline & \multicolumn{2}{|l|}{$\alpha$-Conidia } & \multicolumn{2}{|l|}{$\beta$-Conidia } \\
\hline & Range & Average & Range & Average \\
\hline 1 & $(4.6-7.36$ to $1.84-3.68) \mu$ & $6.44 \times 3.10 \mu$ & $(25.76-55.2$ to $1.84-3.68) \mu$ & $36.69 \mu$ \\
\hline 2 & $\begin{array}{l}(4.6-7.36 \text { to } 2.76-3.68 \\
) \mu\end{array}$ & $5.63 \times 3.53 \mu$ & $(25.76-44.16$ to $1.84-3.68) \mu$ & $36.80 \mu$ \\
\hline 3 & $(4.6-7.36$ to $1.84-3.68) \mu$ & $5.98 \times 3.13 \mu$ & $(22.08-55.2$ to $1.84-3.68) \mu$ & $43.24 \mu$ \\
\hline 4 & $\begin{array}{l}(4.6-7.36 \text { to } 1.84-3.68 \\
) \mu\end{array}$ & $5.98 \times 2.94 \mu$ & $(36.8-40.48$ to $1.84-3.68) \mu$ & $38.03 \mu$ \\
\hline 5 & $\begin{array}{l}(4.6-7.36 \text { to } 1.84-2.76 \\
\mu\end{array}$ & $6.72 \times 1.99 \mu$ & $(25.76-51.52$ to $1.84-3.68) \mu$ & $36.06 \mu$ \\
\hline
\end{tabular}

Table 4. Measurement of pycnidia of Phomopsis vexans .

\begin{tabular}{|c|c|c|}
\hline \multirow{2}{*}{$\begin{array}{c}\text { Grouping } \\
\text { No. }\end{array}$} & \multicolumn{2}{|c|}{$\begin{array}{c}\text { Size of Pycnidia } \\
\text { (Length } \mathrm{x} \text { breadth) }\end{array}$} \\
\cline { 2 - 3 } & Range & Average \\
\hline 1 & $(216.96-284.76$ to $101.7-149.16) \mu$ & $246.34 \mu \times 144.64 \mu$ \\
\hline 2 & $(271.2-589.86$ t o $305.1-637.32) \mu$ & $429.4 \times 258.38 \mu$ \\
\hline 3 & $(462.68-490.42$ to $237.3-271.2) \mu$ & $474.6 \times 255.38 \mu$ \\
\hline 4 & $(372.9-474.6$ to $271.2-406.8) \mu$ & $406.8 \times 327.7 \mu$ \\
\hline 5 & $(271.2-406.8$ to $216.96-305.1) \mu$ & $323.18 \times 264.42 \mu$ \\
\hline
\end{tabular}




\section{REFERENCES}

Chinenye, N. 1974. Occurrence of Phomopsis on Maize (Zea mays). Plant Dis. Reptr. 58(5): 416.

Gratz, L.O. 1942. The perfect stage of Phomopsis vexans . Phytopathology 32: 540-542.

Islam, M.M.; Islam, M.R.; Stevens, C.; Meah, M.B. and Miah, M.A.T. 2004a. Molecular characterization of Phomopsis vexans in the core eggplant producing areas of Bangladesh. Sixth Biennial Con. Bangladesh Phytopathol. Soc. p. 2. Dated. 29 July 2004.

Islam, M.R.; Islam, M.R.; Islam, M.M. and Meah, M.B. 2004b. Physiological features of different isolates of Phomopsis vexans isolated from different eggplant cultivars of major growing regions of Bangladesh. Sixth Biennial Con. Bangladesh Phytopathol. Soc. p. 2. Dated. 29 July 2004.

Meah, M. B. 2003. Development of an integrated approach for management of Phomopsis blight and fruit rot of eggplant in Bangladesh. Annual research report (2002-2003). Department of Plant Pathology Bangladesh Agricultural University, Mymensingh, Bangladesh. pp. 43-44.

Punithalingam, E. and Holliday, P. 1972. CMI Description of Pathogenic Fungi and Bacteria no. 338. Commonwealth Mycological Institute. Ferry Lane, Kew, Surrey. England.

Sharma, N.D. and Agarwal, G.P. 1973. Fungi causing plant disease at Jabalpur (M.P.): Some fungi new to India. Indian Phytopath. 26:545.

Singh, R.S. 1992. Diseases of Vegetable crops, second edn. Oxford and IBH publishing company Pvt. Ltd. New Delhi, Bombay, Calcutta.pp. 119-121.

Sugha, S. K.; Narender, K.; Suman, K.; Kaushal, N.; and Kumar, S. 2002. Factors affecting development of Phomopsis fruit rot of eggplant. Indian Phytopath. 55(1): pp. 26-29.

Walker, J. C. 1952. Diseases of vegetable crops. Mc Graw Hill Company. Inc. New York. pp. 306-308. 